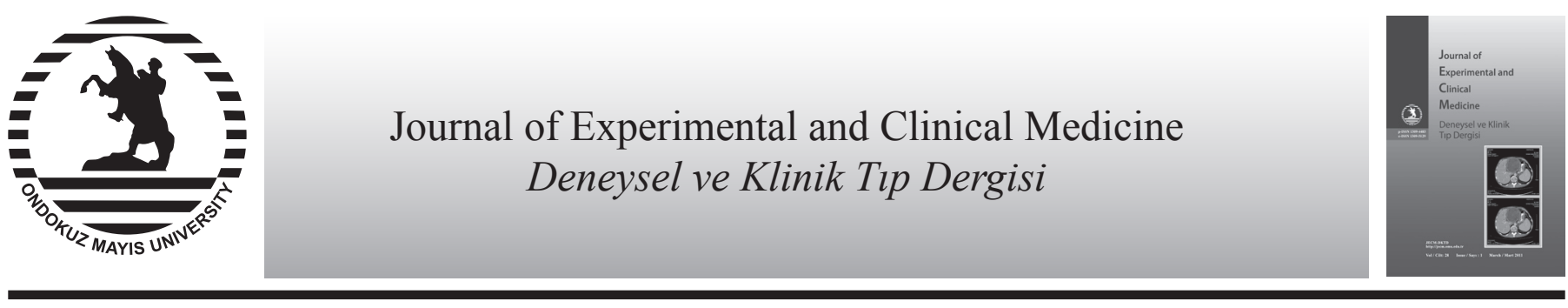

Case Report

\title{
Reversible posterior cerebral artery ischemia related to migraine with aura
}

\author{
Murat Terzi*, Dilek Kasım Yücel, Neslihan Ünal Akdemir \\ Department of Neurology, Medical Faculty, Ondokuz Mayis University Samsun, Turkey
}

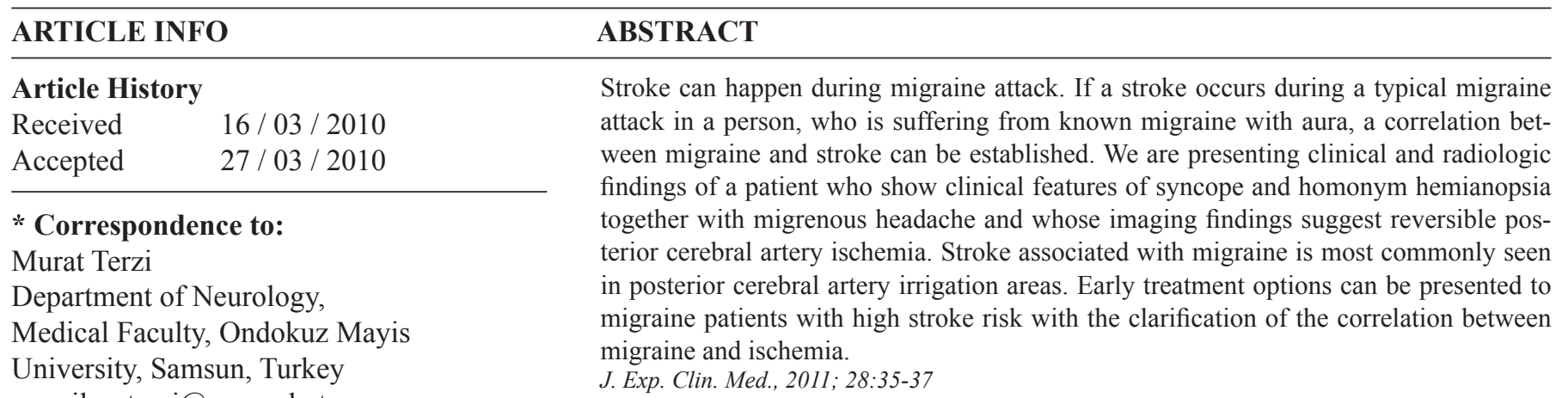

e-mail: mterzi@omu.edu.tr

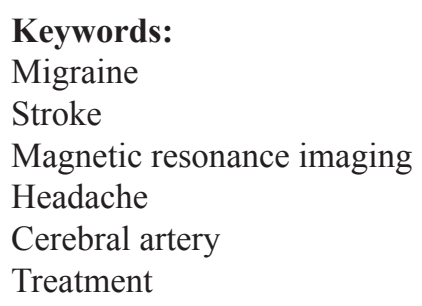

(C) 2011 OMU All rights reserved

\section{Introduction}

Even though migraine is commonly seen, stroke resulting from migraine is somewhat rare. In medical literature, among young patients with stroke, stroke associated with migraine varies between 5-25\% (Bousser and Welch, 2005; Agostoni and Rigamonti, 2007). Stroke can happen during migraine attack or a stroke can cause the migraine attack (Kurth, 2007). Since either migraine or stroke is commonly seen in the society, both conditions can coincidentally co-occur (Kurth, 2007). The risk of stroke is increased even more, especially for female patients over 45 who have migraine with aura if they also smoke and/or use oral contraceptives (Paemeleire, 2009). If a stroke occurs during a typical migraine attack in a person, who is suffering from known migraine with aura, a correlation between migraine and stroke can be established (Bousser and Welch, 2005). However the resulting neurological deficit should be similar to the symptoms seen during previous migraine attacks and all of the ethiological causes that would result in stroke should be excluded.

We are presenting clinical and radiologic findings of a patient who show clinical features of syncope and homonym hemianopsia together with migrenous headache and whose imaging findings suggest reversible posterior cerebral artery ischemia.

\section{Case Report}

A 29 years old, right handed, male patient presented at our clinic with complaints of headache and fainting. The complaints of the patient had occurred 15 days before he came to our clinic. The headaches of the patient who had been suffering from migrenous headaches for 9 years would happen 5-6 times in a month and last for 2-3 days. He would see dark spots in front of his eyes and suffer from a visual problem, which he could not describe, that would last for 10-15 seconds during periods in which the pain aggravates. The headache had started 15 days ago in the morning and he had consumed $500 \mathrm{mg}$ of oral paracetamol. The blood pressure profile at time of symptoms presentation was normal. His pain had been continuing despite being decreased. At noon of the same day he had realized that he could not see objects on his right side. He had fainted 15 minutes later. He had stayed unconscious for about 30 minutes. Contraction, urinary incontinence, foaming at mouth had not occurred. When he had gained consciousness his headache had ceased whereas difficulty to see the objects at his right side had continued. His sight problem had resembled previous headaches however it had been longer and in the form of not seeing right side significantly. He had not had headaches during the 15 day period until he presented at our clinic. At the neurologi- 
cal examination he had homonym hemianopsia on the right. Other neurological evaluations and systematic examination were normal. The patient did not have a history of smoking, alcohol and substance abuse. He has been using paracetamol or naproxen $\mathrm{Na}$ 5-6 times a month for the last 3 years in order to relieve the headaches. He did not have a history of Triptan use. The brain BT imaging taken about 40 hours after the event showed an appearance coherent with hypodens ischemia at the left temporo occipital region, posterior cerebral artery irrigation area (Fig. 1).
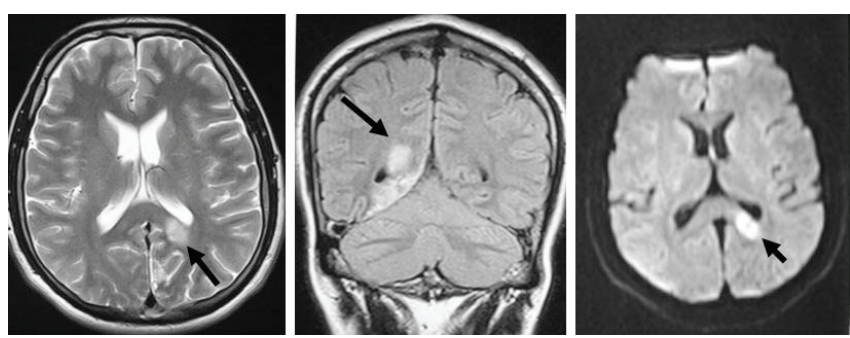

Fig. 1. The brain BT taken on the second day after the event showed an appearance coherent with hypodens ischemia at the left temporo occipital region.

The values of ANA, Anti-DNA, Anticardiolipin, Lupus anticoagulant, homosystein, protein $\mathrm{C}$, protein $\mathrm{S}$, antithrombin 3 which are examined in respect of young stroke were normal. Factor V leiden mutation was negative. Lactic acid was normal. Transthoracic and transesophageal echocardiography, bilateral carotid and vertebral artery doppler examination results were normal. There was not any vascular pathology at brain BT angiography examination.

The diffusion brain MRI taken after 17 days following the event showed coherent with subacute ischemia on left medial occipital cortical surfaces which suggested a significant recovery when compared to previous imaging findings (Fig. 2).

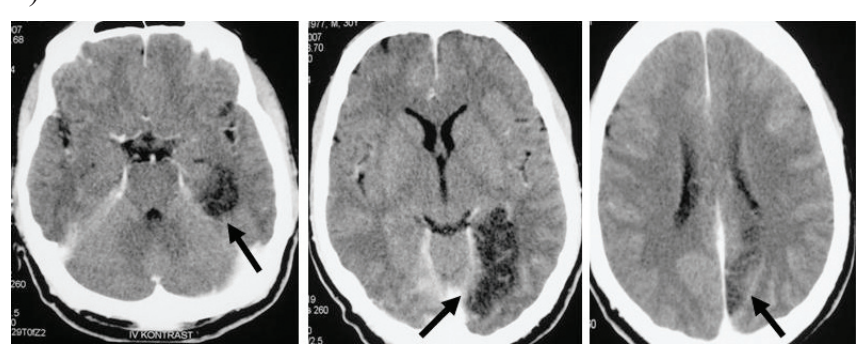

Fig. 2. The diffusion brain MRI taken after 17 days the following the event showed coherent with subacute ischemia on left medial occipital cortical surfaces.

At the brain BT which was taken after 20 days following the event the ischemic change on the left posterior cerebral artery irrigation area was close to full recovery (Fig. 3).
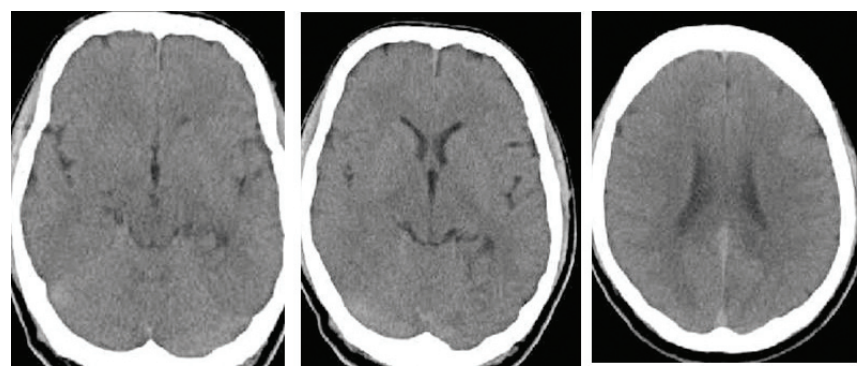

Fig. 3. Brain BT normal after 20 days folowing the event.
In the 3 week period following the event the patient did not have any headaches even though he did not undergo prophylactic treatment. The patient who was administered calcium channel blocker for prophylactic purposes did not have any complaints apart from mild headaches one or two times a month for three months that would last 1-2 hours and did not require him to use analgesic. The homonym hemianopsia on the right had been fully cured at the examination done on the first month following the event.

\section{Discussion}

Stroke associated with migraine is most commonly seen in posterior cerebral artery irrigation areas (Agostoni and Rigamonti, 2007). Frontal and medial cerebral artery ischemia associated with migraine has also been reported (Frigerio et al., 2004; Bousser and Welch, 2005). In our case there were radiological and clinical findings coherent with ischemia on the left posterior cerebral artery irrigation area. The pathogenesis of stroke associated with migraine is not clear. Ischemia is considered to be responsible for the increase in cerebral vasospasm, thrombocyte aggregation (Marshall et al., 2007; Tietjen, 2009). If stroke develops during a typical migraine attack in a person with known migraine with aura, a correlation between migraine and stroke can be established (Bousser and Welch, 2005). However the resulting neurological deficit should be similar to the symptoms seen during previous migraine attacks and all of the ethiological causes that would result in stroke should be excluded. In our case there was a history of migrenous headache and also on the day of event there was headache. In addition, in the previous headache attacks there were short term neurological manifestations similar to homonym hemianopsia which occurred immediately after the current event. Apart from migraine, embolic or thrombotic risk factors which would explain the ischemia in our patient could not be found. There was not any vascular pathology at brain BT angiography.

Changes in brain blood flow up to $40 \%$ during migraine attacks have been showed previously by using analysis such as PET, SPECT, Doppler (Bousser and Welch, 2005; Morelli et al, 2007). In studies and clinical observations carried out on migraine patients the subclinical white matter changes can be commonly seen in brain MRI (Marshall et al., 2007). However the number of cases in which reversible ischemic change can be shown by imaging findings are somewhat low (Resnick et al., 2006). Our case showed a significant left posterior cerebral artery ischemia on the brain BT carried out on the second day of the event, a partial recovery on brain MRI carried out on the 17th day after the event and a full recovery on the brain carried out on the 20th day after the event. A full recovery was observed for the homonym hemianopsia at the neurological examination performed during the same period of the radiological recovery.

There are studies that show vasoconstriction and vasodilatation in migraine and cerebral ischemia associated with vasoconstriction might occur (Bousser and Welch, 2005; Morelli et al., 2007). The short recovery in the clinical and radiological findings of our case can be explained by the reversible vasospasm at the posterior cerebral artery. Another manifestation is that clinical and radiological findings that point to vasogenic oedema on extended aura and ischemia associated with permanent neuronal suppression can be seen (Agostoni and Rigamonti, 2007; Kurth, 2007). A full recovery in neuro- 
radiological findings in correlation with clinical features as in our case suggest for possible reversible neuronal inflammatory pathology in typical migraine with aura.

There are no indicators that show stoke risk in migraine patients. Major artery ischemia associated with migraine and the physiopathology of the subclinical changes in the white matter are not fully known. Studies that clarify the nature of ischemic changes in migraine and their correlation with clinical features are needed. Early treatment options can be presented to migraine patients with high stroke risk with the clarification of the correlation between migraine and ischemia.

\section{REFERENCES}

Agostoni, E., Rigamonti, A., 2007. Migraine and cerebrovascular disease. Neurol. Sci. 28, 156- 160.

Bousser, M.G., Welch, K.M., 2005. Relation between migraine and stroke. Lancet Neurol. 4, 533- 542.

Frigerio, R., Santoro, P., Ferrarese, C., Agostoni, E,. 2004. Migrainous cerebral infarction: case reports. Neurol. Sci. $25,300-301$.

Kurth, T., 2007. Migraine and ischaemic vascular events. Cephalalgia. 27, 965-975.

Marshall, N., Maclaurin, W.A., Koulouris, G., 2007. MRA captures vasospasm in fatal migrainous infarction. Headache. 47, $280-283$.

Morelli, N., Gori, S., Mancuso, M., Murri, L., 2007. Basilar artery fenestration in a woman with basilar-type migraine. Neurol. Sci. 28, 11617.

Paemeleire, K., 2009. Brain lesions and cerebral functional impairment in migraine patients. J. Neurol. Sci. 283, 134-136.

Resnick, S., Reyes-Iglesias, Y., Carreras, R., Villalobos, E., 2006. Migraine with aura associated with reversible MRI abnormalities. Neurol. 66, 946-947.

Tietjen, G.E., 2009. Migraine as a systemic vasculopathy. Cephalalgia. 29, 987-996. 